\title{
Photoelectron Imaging of Helium Droplets Doped with Xe and Kr Atoms ${ }^{\dagger}$
}

\author{
Chia C. Wang, Oleg Kornilov, Oliver Gessner, Jeong Hyun Kim, ${ }^{\S}$ Darcy S. Peterka, $₫$ and \\ Daniel M. Neumark*
}

Department of Chemistry, University of California, Berkeley, California 94720, and Chemical Sciences Division, Lawrence Berkeley National Laboratory, Berkeley, California 94720

Received: March 17, 2008; Revised Manuscript Received: June 4, 2008

\begin{abstract}
Helium droplets doped with $\mathrm{Xe}$ and $\mathrm{Kr}$ atoms were photoionized by using VUV synchrotron radiation from the Advanced Light Source and the resulting photoelectron images were measured. A wide range of He droplet sizes, photon energies, and dopant pick-up conditions was investigated. Significant ionization of dopants was observed at $21.6 \mathrm{eV}$, the absorption maximum of $2 \mathrm{p}^{1} \mathrm{P}_{1}$ electronic excited state of He droplets, indicating an indirect ionization mechanism via excitation transfer. The photoelectron images and spectra reveal multiple photoionization mechanisms and pathways for the photoelectrons to escape the droplet. Specifically, they show sets of sharp peaks assigned to two mechanisms for Penning ionization of the dopant by $\mathrm{He}^{*}$ in which the photoelectrons leave the droplet with no detectable energy loss, a broad, intense feature representing electrons that undergo significant energy loss, and a small amount of ultraslow electrons that may result from electron trapping at the droplet surface. The droplet-size dependence of the broad, intense feature suggests the development of the conduction band edge in the largest droplets seen here $(\langle N\rangle \approx 250,000)$.
\end{abstract}

\section{Introduction}

This work presents a study of photoionization of He nanodroplets doped with $\mathrm{Xe}$ and $\mathrm{Kr}$ atoms and the resulting photoelectron dynamics. It is motivated by our desire to understand the mechanism of photoionization in doped $\mathrm{He}$ droplets, as well as the transport and escape of the photoelectrons produced by this process. By doing so, we hope to gain insight into the coupling between charged particles and the He droplet environment. The experiments are performed by using tunable vacuum ultraviolet (VUV) synchrotron radiation to ionize the droplets and photoelectron imaging to analyze the resulting photoelectrons.

He nanodroplets have proved to be a novel and versatile spectroscopic environment. ${ }^{1}$ It has been demonstrated experimentally $^{2}$ and theoretically ${ }^{3}$ that cold He droplets with as few as $60 \mathrm{He}$ atoms exhibit superfluidity, paving the way toward understanding this macroscopic quantum phenomenon at the atomic level. Owing to the ability of He droplets to pick up foreign species, first demonstrated in the early 1990s, ${ }^{4,5}$ there has been much interest in their application as an ultracold $(0.37$ $\mathrm{K}$ ) and weakly interacting medium for performing rotational, vibrational, and electronic spectroscopy on droplets doped with atoms, molecules, and clusters. ${ }^{6-10}$ Through their capability to dissipate energy efficiently, He droplets can also serve as a cryogenic platform to synthesize unusual complexes by trapping target species in local minima. ${ }^{11-13}$

Considerably stronger interactions with the droplet environment are expected for charged particles, both ions and electrons, and many experiments have been performed to unravel these interactions. One class of experiments has focused on the remarkable fact that electrons can attach and be incorporated

\footnotetext{
$\dagger$ Part of the Stephen R. Leone Festschrift.

* Corresponding author. Email: dneumark@berkeley.edu.

$\S$ Current address: School of Chemical and Biological Engineering, College of Engineering, Seoul National University, Seoul 151-744, Korea

$¥$ Current address: Center for Neurobiology and Behavior, Biological Sciences Department, Columbia University, New York, New York.
}

into He droplets, ${ }^{14}$ forming electron bubbles within droplets analogous to those observed in bulk liquid He. ${ }^{15,16}$ In addition, there have been numerous experiments on electron impact ${ }^{17-23}$ and photoionization ${ }^{24-31}$ of both pure and doped He droplets. Many of these experiments and the accompanying theory ${ }^{32-35}$ have focused on the ion yield and mass spectrometry of the ionization products as a function of electron impact or photon energy, with the goal of elucidating the mechanism of ionization and the response of the droplet to having an ion (or ion cluster) formed within. The other class of experiments, including the present work, centers on photoelectron spectroscopy, which yields further insights into the ionization mechanism and the extent to which the photoelectron generated by ionization interacts with the droplet atoms.

In the first photoionization experiment on He droplets, carried out by Fröchtenicht et al., ${ }^{24}$ pure droplets and droplets doped with $\mathrm{SF}_{6}$ were ionized with tunable VUV synchrotron radiation. The ionization threshold for pure droplets was found to be around $23 \mathrm{eV}$, significantly lower than $\mathrm{IP}(\mathrm{He})=24.6 \mathrm{eV}$. The lower threshold in droplets was attributed to the formation of $\mathrm{He}_{2}{ }^{+}$or larger cation clusters within the droplet. In the doped droplets, a sharp peak in the $\mathrm{SF}_{5}{ }^{+}$ion yield was seen at 21.6 $\mathrm{eV}$. This energy coincides with the lowest optically allowed electronic excitation in pure He droplets, as first observed by Joppien et al. ${ }^{36}$ via fluorescence excitation; this transition was assigned to the droplet analogue of the $2 \mathrm{p}{ }^{1} \mathrm{P}_{1}-1 \mathrm{~s}{ }^{1} \mathrm{~S}_{0}$ transition in the atomic $\mathrm{He}$ at $21.2 \mathrm{eV}$, blue-shifted and broadened in the droplet owing to collective interactions in the excited state. ${ }^{37}$ The photoionization experiments showed that the $\mathrm{SF}_{6}$ dopant was ionized via an indirect mechanism in which the He droplet was electronically excited and excitation transfer from the surrounding droplet ionized the dopant, that is, an intracluster Penning ionization mechanism. A similarly enhanced ion yield at $21.6 \mathrm{eV}$ was seen by Kim et al. ${ }^{26}$ and Peterka et al. ${ }^{38}$ in photoionization experiments on He droplets doped with rare gas atoms $(\mathrm{Rg})$ and $\mathrm{SF}_{6}$, respectively. 
Further insight into the mechanisms of ionization and electron transport in He droplets is provided by photoelectron spectroscopy experiments in which the electron kinetic energy (eKE) and angular distribution are determined. Photoelectron imaging of pure He droplets was studied by Peterka et al. ${ }^{25,39}$ between 22.5 and $28 \mathrm{eV}$. At photon energies below the atomic $\mathrm{He}$ ionization potential, between 23.0 and $24.6 \mathrm{eV}$, the photoelectron spectrum primarily comprised ultraslow electrons $(<1 \mathrm{meV}$ kinetic energy). Although these slow electrons were also seen at photon energies above the ionization potential of atomic $\mathrm{He}$, $24.6 \mathrm{eV} \leq h v \leq 28.0 \mathrm{eV}$, the photoelectron spectrum at those energies was dominated by much faster electrons in the same kinetic energy range as that for atomic $\mathrm{He}$ but shifted and broadened toward higher energy by as much as $0.5 \mathrm{eV}$. The fast electrons were attributed to direct ionization of the He atoms in the droplet. The shifting and broadening were explained by a dimer model in which vertical photoionization accesses the attractive $\mathrm{He}_{2}{ }^{+}$potential energy curve between two nearest neighbor $\mathrm{He}$ atoms. In contrast, the slow electrons were attributed to a complex mechanism involving (i) formation of an electron bubble within the droplet, (ii) temporary trapping of the electron at the surface of the cluster via the attractive interaction with the positively charged cluster, ${ }^{40,41}$ and (iii) ejection of the electron via vibrational autoionization.

Peterka et al. ${ }^{38}$ measured photoelectron images of droplets doped with $\mathrm{SF}_{6}$ at a photon energy of $21.8 \mathrm{eV}$; these were the first photoelectron spectra obtained by indirect dopant ionization. The spectra were similar to those of gas phase $\mathrm{SF}_{6}$, although detailed comparisons were complicated by the fact that $\mathrm{SF}_{6}$ undergoes dissociative ionization to $\mathrm{SF}_{5}{ }^{+}+\mathrm{F}$; therefore, all the spectral features were quite broad. However, there appeared to be an underlying continuous contribution to the droplet spectrum that was most noticeable below $2 \mathrm{eV}$.

In complementary studies, direct ionization of dopants in $\mathrm{He}$ droplets has been observed by several groups via resonanceenhanced multiphoton ionization. ${ }^{27-30,42}$ Meiwes-Broer and co-workers ${ }^{29,42}$ probed excited-state relaxation of silver clusters embedded in He droplets by photoelectron spectroscopy, whereas Drabbels and co-workers ${ }^{27,28}$ measured photoelectron images of droplets doped with aniline and silver atoms. The signature of direct ionization, as exemplified by the experiment of Loginov et al. ${ }^{27}$ on droplets doped with aniline, is an ion yield spectrum the features of which, although shifted and broadened, correspond with those of the $S_{1}-S_{0}$ transition in their gas phase counterpart. Their photoelectron imaging experiments showed that photoelectron features from the doped droplets were shifted by $200-1000 \mathrm{~cm}^{-1}(0.025-0.124 \mathrm{eV})$ toward higher kinetic energy compared to the isolated gas phase species. This effect is attributed to a lowering ionization potential of the dopant in the droplet from stabilization of the positively charged species by the polarizable He solvent. In addition, the photoelectron spectrum showed asymmetric broadening toward lower kinetic energy, suggesting some electron energy loss during escape from the droplet.

This set of photoelectron spectroscopy experiments points to complex ionization phenomena in pure and doped $\mathrm{He}$ droplets. Some of the effects, such as the shifting of peaks toward higher eKE from direct ionization of either He atoms in the droplet or the droplet dopant, are explained in terms of simple models of ionization which involve little or no interaction of the droplet atoms with the photoelectron. On the other hand, the very slow photoelectrons seen in the synchrotron experiments point to strong final state interactions in which the eKE is strongly perturbed by the droplet environment. The droplet

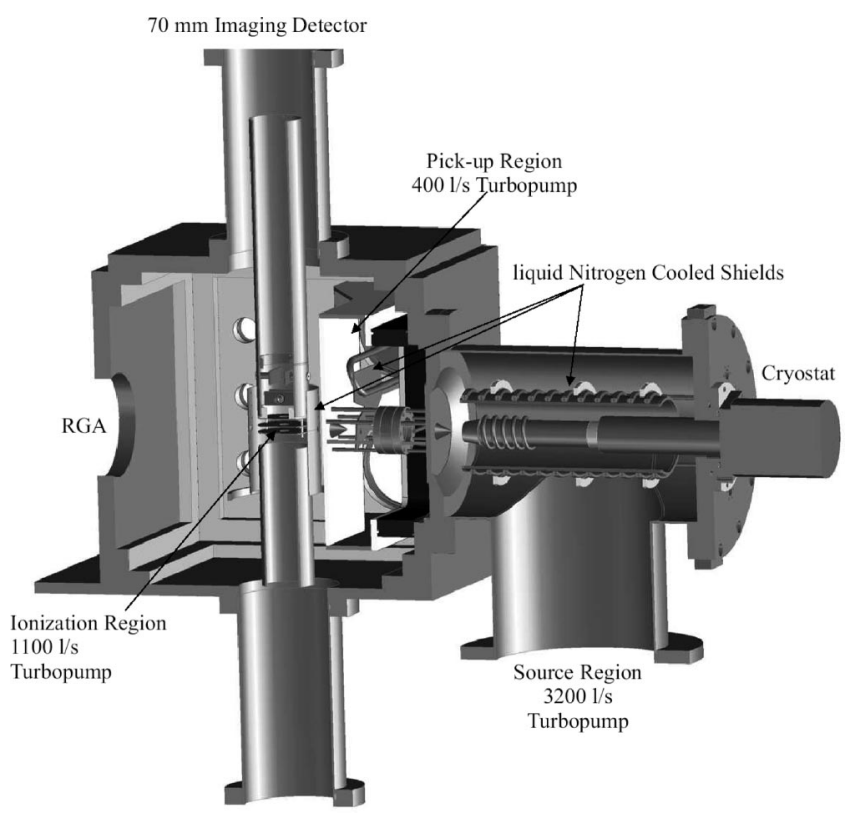

Figure 1. Schematic view of He-droplet instrument.

size in all these experiments is in the range of $10^{4}-10^{5}$ atoms, and it is perhaps surprising that except for the experiments on pure droplets below $\mathrm{IP}(\mathrm{He}),{ }^{25}$ the photoelectron signal is not dominated by strong final state interactions, given the propensity of free electrons in bulk liquid He to form electron bubbles with very low mobility. ${ }^{16,43,44}$ Moreover, numerous studies have shown that there is a barrier (or the conduction band edge of liquid $\mathrm{He}$, relative to vacuum) on the order of $1 \mathrm{eV}$ for electron injection into both bulk liquid $\mathrm{He}^{15,45-49}$ and He droplets, ${ }^{14,50-52}$ and one might expect to see manifestations of this barrier for electrons ejected from droplets.

These considerations motivate the work presented here, in which we perform photoelectron imaging experiments on droplets with up to $250000 \mathrm{He}$ atoms doped with either Xe or $\mathrm{Kr}$. Our studies focus on indirect ionization in which the droplets are electronically excited to the $2 \mathrm{p}{ }^{1} \mathrm{P}_{1}$ state at $21.6 \mathrm{eV}$, leading to Penning ionization of the dopant. The relative simplicity of these dopants enables one to probe the mechanisms of ionization and electron transport in He droplets in considerable detail. Our experiments yield evidence of four distinct mechanisms for photoelectron ejection. Specifically, we observe two Penning ionization channels, one from $\mathrm{He}^{*}\left(2 \mathrm{p}{ }^{1} \mathrm{P}_{1}\right)+\mathrm{Rg}$ and one from $\mathrm{He}^{*}\left(2 \mathrm{~s}{ }^{1} \mathrm{~S}_{0}\right)+\mathrm{Rg}$, a broad feature that is suggestive of electron ejection from the droplet analogue of the liquid helium conduction band, and ultraslow electrons analogous to previous photoelectron imaging experiments on pure He droplets. ${ }^{25,39}$

\section{Experiment}

Experiments were carried out on the Chemical Dynamics Beamline at the Advanced Light Source (ALS). The apparatus is illustrated in Figure 1. A continuous He-droplet beam was generated by sending ultrahigh-purity He gas (research grade, $99.9999 \%$ ) at 20 bar through a $5 \mu \mathrm{m}$ diameter aperture mounted on the second stage of a closed-cycle He refrigerator (ARS DE202NFF). The high-pressure He gas, after expansion through the small orifice, underwent adiabatic and evaporative cooling and condensed into droplets. The droplet-size distribution was adjusted by varying the source temperature, $T_{\mathrm{s}}$, between 9 and $19 \mathrm{~K}$; the source temperature was monitored by a silicon diode (Lakeshore DT 470-SD11) and controlled by a temperature 
controller (LakeShore LS331). The droplet size distribution under these source conditions has been characterized previously in scattering deflection experiments by Lewerenz et al. ${ }^{53}$ The resulting He-droplet beam subsequently entered a pickup chamber after passing through a $1.5 \mathrm{~mm}$ diameter skimmer.

Dopants were picked up and embedded in He droplets passing through the pickup cell, which was $5 \mathrm{~cm}$ in length with openings of $2.5 \mathrm{~mm}$ in diameter in both sides. $\mathrm{Kr}$ or Xe (research grade) was introduced into the pickup cell through a variable rate precision leak valve (Varian Model 951-5106) at room temperature. A microion gauge (Granville-Phillips 355) monitored the pressure in the pickup cell. The pickup pressure was varied between $10^{-6}$ torr and $10^{-4}$ torr and calibrated according to the ion gauge gas correction factor relative to nitrogen. The average number of dopant atoms picked up by He droplets follows a Poisson distribution, ${ }^{54}$ and although we were most interested in conditions under which single pickup occurred, the effects of multiple pickup were also investigated. After the pickup chamber, the doped He-droplet beam entered the main chamber and intersected the VUV synchrotron radiation beam at the interaction region. The VUV radiation was generated from the U-10 undulator of the ALS. A 3 m off-plane Eagle monochromator fitted with a 600 line $/ \mathrm{mm}$ Ir-coated grating provided $\sim 10^{13}$ photons/s in the range of $20-26 \mathrm{eV}$ with $18 \mathrm{meV}$ bandwidth.

Photoelectrons were collected and analyzed by using velocity map imaging. ${ }^{55}$ A stack of electron optics accelerated the ejected photoelectrons into a field-free flight tube of $60 \mathrm{~cm}$ in length. In order to detect electrons of high kinetic energy $(\sim 10 \mathrm{eV})$, an electric field of $2.5 \mathrm{kV} / \mathrm{cm}$ was applied in the interaction region for electron extraction. After expansion in the flight tube, photoelectrons were detected by an assembly of $75 \mathrm{~mm}$ diameter chevron-mounted multichannel plates coupled to a phosphor screen (APD 3075FM-P47). A CCD camera (DALSA 1M30) was mounted outside the vacuum chamber to image the phosphor screen. The projected 2D images of ejected photoelectrons were converted to 3D distributions by using the basis set expansion (BASEX) Abel transformation method developed by Dribinski et al. ${ }^{56}$

\section{Results}

Figure 2 shows 2D photoelectron images of Xe-doped $\mathrm{He}$ droplets taken at $21.6 \mathrm{eV}$ (Figure 2a) and $20.6 \mathrm{eV}$ (Figure 2b) under the same source (base He pressure 20 bar, source temperature $\left.T_{\mathrm{s}}=13 \mathrm{~K},\langle N\rangle \approx 8000\right)$ and pickup conditions $\left(\sim 5.5 \times 10^{-6}\right.$ torr $\mathrm{Xe}$ in the pickup cell). At these conditions, droplets pick up on average one dopant atom. The photon energies in Figure 2a,b correspond to maximum and near-zero absorption in pure He droplets, respectively. ${ }^{36}$ The photoelectron image of atomic Xe (Figure 2c) is also shown for comparison. Figure $2 \mathrm{c}$ was recorded by sending $\mathrm{Xe}$ gas $\left(\sim 10^{-3}\right.$ torr $)$ into the pickup cell while the He-droplet beam was blocked by a retractable beam flag. Gaseous Xe effused into the interaction region from the pickup chamber via a $2 \mathrm{~mm}$ skimmer that connects these two differential regions.

In the atomic Xe image in Figure 2c, two sharp rings with a strongly anisotropic angular distribution are observed, corresponding to the ${ }^{2} \mathrm{P}_{3 / 2}$ and ${ }^{2} \mathrm{P}_{1 / 2}$ spin- orbit states of $\mathrm{Xe}^{+}$that are split by $1.307 \mathrm{eV} .^{57}$ The photoelectron image of Xe-doped droplets in Figure 2a is quite different. The outer rings are less intense and more isotropic. There is a broad feature at smaller radius with no definite structure. Finally, there is a bright center spot. In contrast to Figure $2 \mathrm{a}$, the image of Xe-doped droplet taken at $20.6 \mathrm{eV}$ (Figure 2b) exhibits a smaller total electron (a)

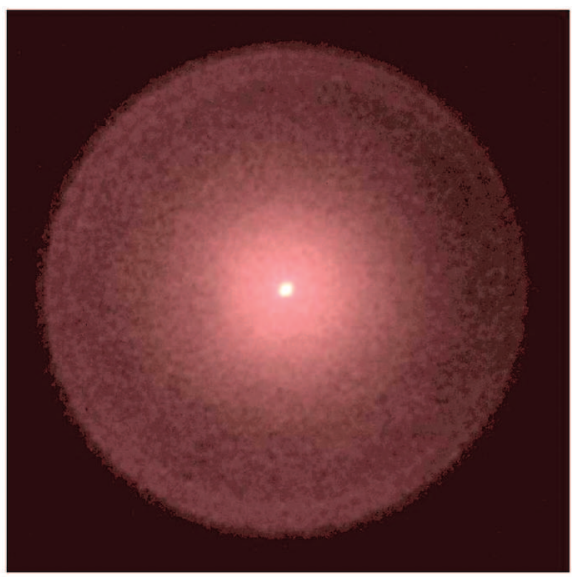

(b)

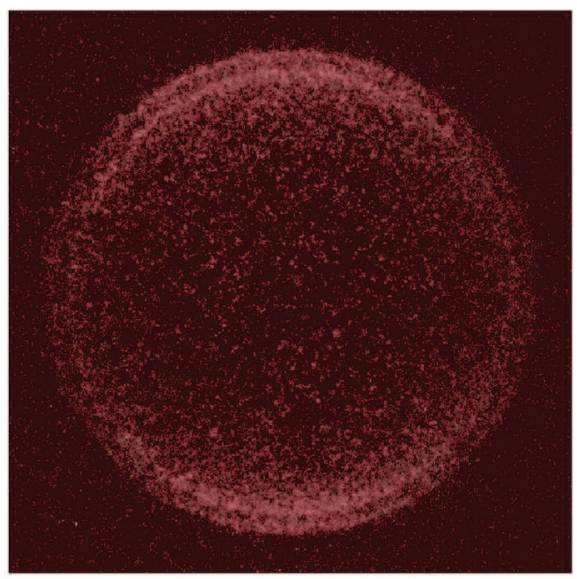

(c)

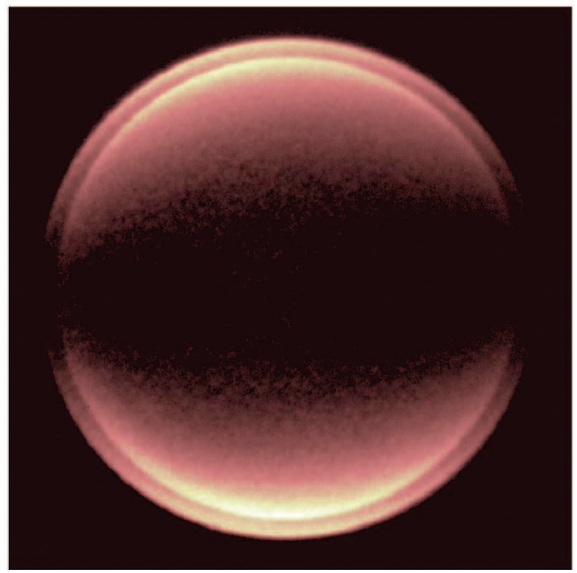

Figure 2. Photoelectron images of (a) Xe doped in He droplets at $21.6 \mathrm{eV}$, (b) Xe doped in He droplets at $20.6 \mathrm{eV}$, and (c) atomic $\mathrm{Xe}$ at $21.6 \mathrm{eV}$.

yield (TEY), by about a factor of 20, and in fact strongly resembles the atomic image in Figure 2c.

Further information can be obtained by photoelectron kinetic energy spectra obtained from the images. Figure 3 shows photoelectron spectra of He droplets doped with $\mathrm{Kr}$ (Figure 3a) and $\mathrm{Xe}$ (Figure 3b), taken at droplet source and pickup conditions similar to those in Figure 2. Each panel shows spectra of doped droplets taken at $21.6 \mathrm{eV}$ (black line) and $20.6 \mathrm{eV}$ (dark gray line) together with the photoelectron spectrum of the bare dopant atom at $21.6 \mathrm{eV}$ (light gray line). The spectra taken at $20.6 \mathrm{eV}$ have been shifted toward higher eKE by $1 \mathrm{eV}$ to facilitate comparison with the spectra taken at $21.6 \mathrm{eV}$. The spectra at $20.6 \mathrm{eV}$ are essentially identical with those of the 


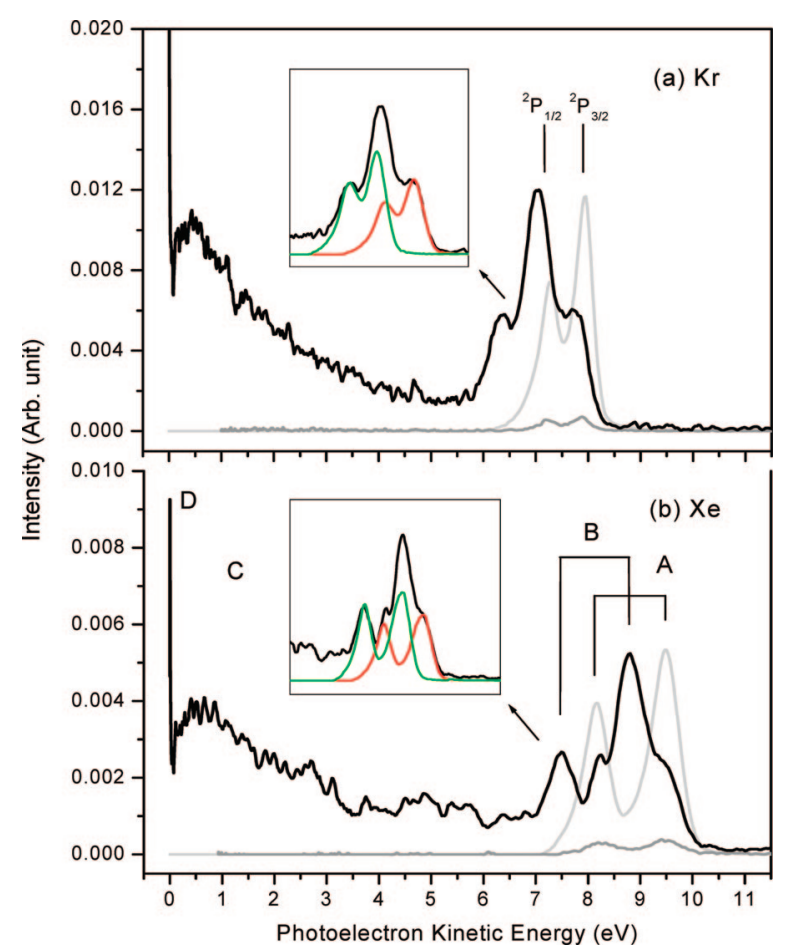

Figure 3. (a) Photoelectron spectra of $\mathrm{Kr}$-doped He droplets at 21.6 $\mathrm{eV}$ (black) and $20.6 \mathrm{eV}$ (gray) and of atomic $\mathrm{Kr}$ at $21.6 \mathrm{eV}$ (light gray). (b) Photoelectron spectra of Xe-doped He droplets at $21.6 \mathrm{eV}$ (black) and $20.6 \mathrm{eV}$ (gray) and of atomic $\mathrm{Xe}$ at $21.6 \mathrm{eV}$ (light gray). Note that feature $\mathrm{D}$ in panel a is clipped for view purpose.

bare rare gas atoms and are attributed to direct ionization of $\mathrm{Kr}$ or Xe atoms that have effused from the pickup cell into the interaction region rather than to ionization of doped droplets. The remaining discussion thus focuses on doped droplets ionized at $21.6 \mathrm{eV}$.

At $21.6 \mathrm{eV}$, droplets doped with $\mathrm{Kr}$ and $\mathrm{Xe}$ show several features. First, there is a set of partially resolved peaks at high eKE, between 6-8 eV for $\mathrm{Kr}$ and 7-10 eV for Xe. As discussed below, these peaks appear to be two overlapping features labeled $\mathrm{A}$ and $\mathrm{B}$. For both $\mathrm{Xe}$ - and $\mathrm{Kr}$-doped droplets, there is a broad, lower energy feature, labeled feature $\mathrm{C}$, that rises with decreasing eKE starting around 5-6 eV, peaking around $1 \mathrm{eV}$, and falling off at lower eKE. Finally, there is a sharp feature, feature $\mathrm{D}$, at very low energies, peaking around $1.0 \mathrm{meV}$. This feature corresponds to the bright center spot seen in the image in Figure 2 a.

The nature of features $\mathrm{A}$ and $\mathrm{B}$ is best seen from the $\mathrm{Xe}$ doped droplet spectrum in Figure $3 \mathrm{~b}$. There are two shoulders that line up well with the transitions to the ${ }^{2} \mathrm{P}_{3 / 2}$ and ${ }^{2} \mathrm{P}_{1 / 2}$ spin-orbit states of $\mathrm{Xe}^{+}$at 9.47 and $8.16 \mathrm{eV}$, respectively. There are two more peaks, centered at 8.82 and $7.51 \mathrm{eV}$ that correspond to shifting the pair of atomic transitions toward lower eKE by $0.65 \pm 0.02 \mathrm{eV}$, as obtained by fitting all four peaks to Gaussian profiles. These shifted peaks have about the same width $(0.5 \mathrm{eV})$ as the unshifted peaks. We refer to the unshifted pair of peaks as feature A and the shifted pair as feature B. In the Kr-doped droplet spectrum in Figure $3 \mathrm{a}$, an intense central peak and two side-bands are seen in the $6-8 \mathrm{eV}$ range. However, as shown in the inset in Figure $3 \mathrm{a}$, these three peaks can also be deconvoluted into two unshifted $\mathrm{Kr}^{+}$transitions split by $0.67 \mathrm{eV}$, the spin-orbit splitting of $\mathrm{Kr}^{+}, 57$ and two additional peaks shifted toward lower eKE by the same amount as in Figure 3b. Because the shift is close to the $\mathrm{Kr}^{+}$spin-orbit splitting, the lower energy feature A peak and higher energy feature B

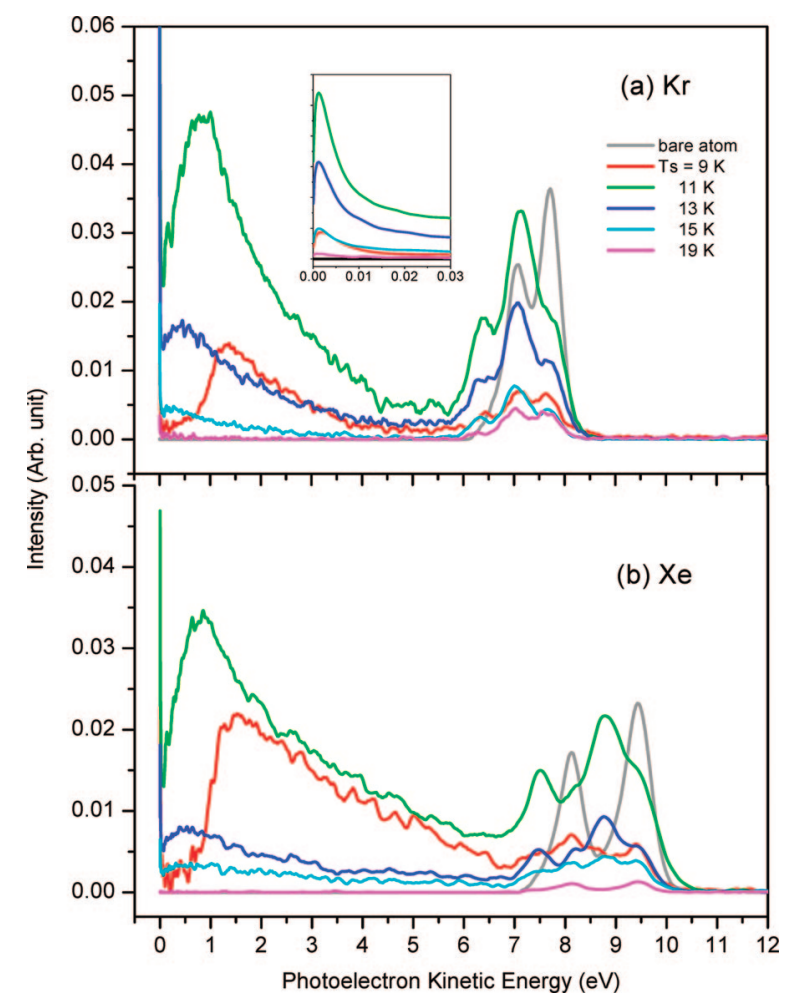

Figure 4. Photoelectron spectra of He droplets doped with (a) $\mathrm{Kr}$ and (b) Xe taken at various source temperatures while keeping the pickup pressure fixed at $\sim 1.2 \times 10^{-5}$ torr. The spectra of bare dopants are also shown for comparison. Note that feature D in panel a is clipped for view purpose.

TABLE 1: Average Droplet Size $\langle N\rangle$ and Mean Density $\rho$ with Respect to the Bulk Liquid Density $\rho_{\text {bulk }}$ at Each Source Temperature $T_{\mathrm{s}}$

\begin{tabular}{clc}
\hline$T_{\mathrm{s}}(\mathrm{K})$ & \multicolumn{1}{c}{$\langle N\rangle$} & $\rho / \rho_{\text {bulk }}$ \\
\hline 9 & 250000 & 0.99 \\
11 & 16000 & 0.85 \\
13 & 8000 & 0.65 \\
15 & 4000 & 0.55 \\
19 & 400 & $<0.4$
\end{tabular}

peak overlap. Hence, in both $\mathrm{Xe}$ and $\mathrm{Kr}$-doped droplets, features $\mathrm{A}$ and $\mathrm{B}$ each comprise a pair of peaks, with the shift between pairs independent of dopant.

Figure 4 shows photoelectron spectra of He droplets doped with $\mathrm{Kr}$ (Figure 4a) and $\mathrm{Xe}$ (Figure 4b) taken at $21.6 \mathrm{eV}$ at several source temperatures, between 9 and $19 \mathrm{~K}$, with the pickup pressure of dopants fixed at $\sim 1.2 \times 10^{-5}$ torr. These spectra are normalized with respect to the TEY. Spectra taken at even lower pickup pressure, $\sim 5.5 \times 10^{-6}$ torr, exhibit fundamentally the same features, except that the TEY is less by a factor of 2, and thus, the signal-to-noise ratio is not as satisfactory as those in Figure 4. Table 1 shows relevant parameters corresponding to each source temperature $T_{\mathrm{s}}$ investigated in the present work, including the mean droplet size $\langle N\rangle$ and the average density $\rho$ with respect to the bulk liquid helium density $\rho_{\text {bulk. }}\langle N\rangle$ is determined from experiments of Lewerenz et al. ${ }^{53}$ under similar expansion conditions; the average density for each droplet size is estimated according to previous experimental work by Harms et al. ${ }^{58}$ The photoelectron spectra of bare dopants are also shown in Figure 4 (gray line) for reference. As the source temperature is lowered from 19 to 11 $\mathrm{K}$, the dopant photoelectron signal increases steadily, presumably because the average droplet size is larger so that the fraction 

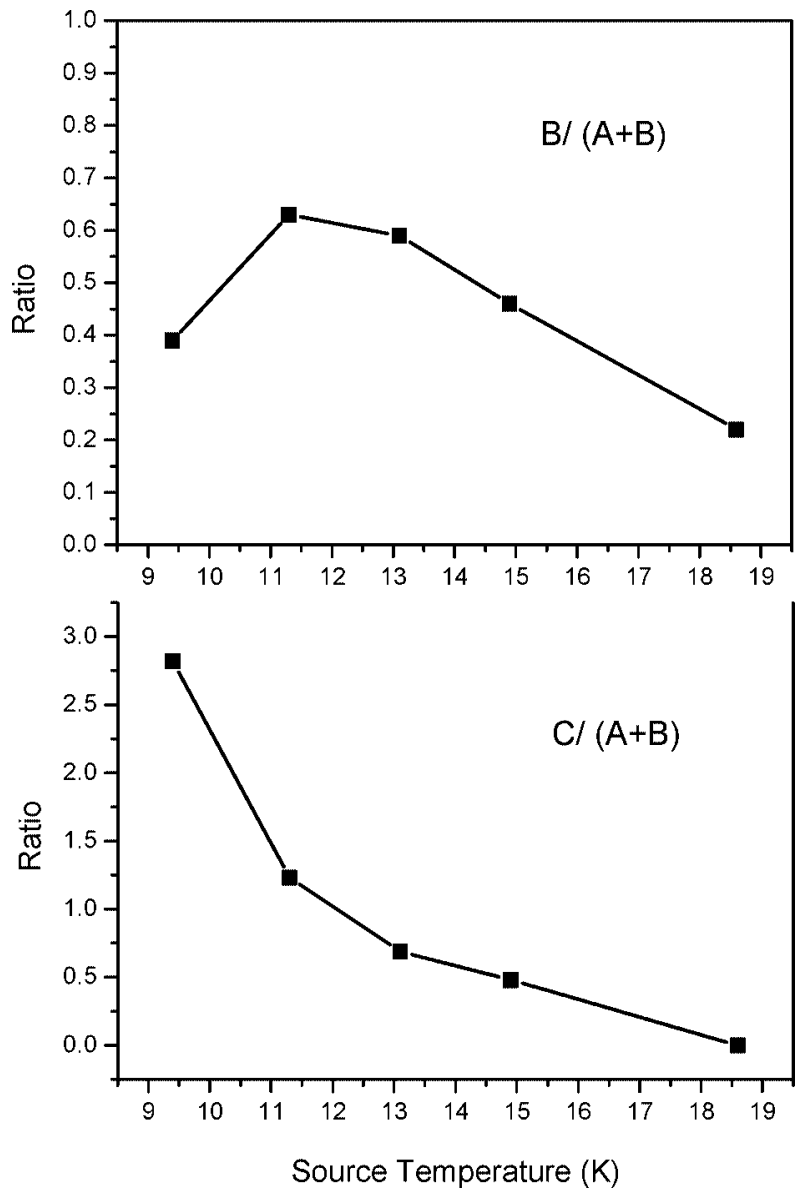

Figure 5. Intensity ratio of $\mathrm{B} /(\mathrm{A}+\mathrm{B})$ and $\mathrm{C} /(\mathrm{A}+\mathrm{B})$ of Xe-doped droplets as a function of source temperature.

of droplets picking up one (or more) dopant atoms increases. The photoelectron signal is considerably lower at $9 \mathrm{~K}$, possibly reflecting the lower efficiency for dopant ionization in the very large clusters formed under these conditions.

The evolution of features $A-D$ with source temperature is apparent in Figure 4. As the temperature is lowered from 19 to $11 \mathrm{~K}$, feature $\mathrm{B}$ becomes progressively more intense relative to feature A but drops noticeably at the lowest temperature, $9 \mathrm{~K}$. The positions of features A and B, however, do not vary with $T_{\mathrm{s}}$. In contrast, the relative intensity of feature $\mathrm{C}$ increases with decreasing $T_{\mathrm{s}}$ for all temperatures in Figure 4, while its intensity maximum shifts to progressively higher eKE. At $9 \mathrm{~K}$, feature $\mathrm{C}$ displays a clear onset at around $1.0 \mathrm{eV}$ and peaks at $1.45 \mathrm{eV}$ $\pm 0.10 \mathrm{eV}$. The inset in Figure 4a shows an enlarged view of feature D. Figure 5 shows the relative intensity ratios $\mathrm{B} /(\mathrm{A}+$ B) (upper panel) and $\mathrm{C} /(\mathrm{A}+\mathrm{B}$ ) (lower panel) of Xe-doped $\mathrm{He}$ droplets at each value of $T_{\mathrm{s}}$. Figure 6 shows the energy of the intensity maximum of feature $\mathrm{C}$ versus the average droplet size radius estimated at various source temperatures.

Figure 7 shows photoelectron spectra of $\mathrm{Kr}$ - and $\mathrm{Xe}$-doped droplets as a function of $T_{\mathrm{s}}$ and pickup cell pressure in order to see how the number of dopant atoms affects the various features in the spectra. The pickup pressures were varied from $1.2 \times$ $10^{-5}$ to $3.3 \times 10^{-5}$ torr. Table 2 lists the average number of dopants picked up at each pickup pressure calculated from a Poisson distribution; ${ }^{54}$ note that at $9 \mathrm{~K}$, where the droplets are very large,${ }^{59}$ multiple dopants are picked up even at the lowest cell pressure. All features in the photoelectron spectra become more intense with increasing pressure, as expected because the average number of dopants per droplet is higher. Feature B,

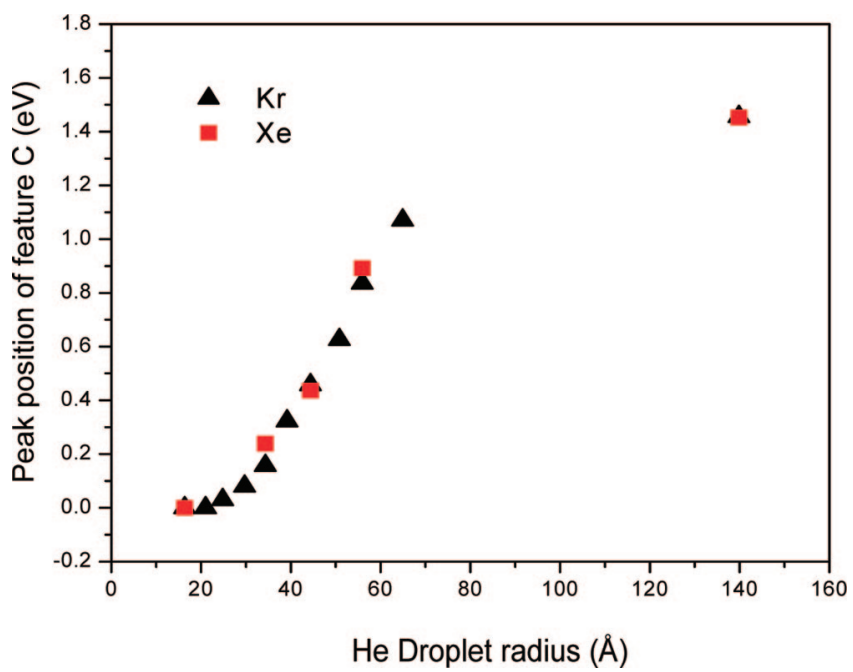

Figure 6. Peak position of feature $\mathrm{C}$ for $\mathrm{Kr}$ and $\mathrm{Xe}$ as a function of $\mathrm{He}$-droplet radius. The peak position of feature $\mathrm{C}$ shifts toward higher $\mathrm{eKE}$ as the droplet size increases and reaches a value of $1.45 \mathrm{eV}$ for the largest droplets.

however, drops in intensity relative to feature $\mathrm{A}$ as the pressure is raised. A small amount of signal at higher kinetic energy than that at which the atomic rare gas peaks is observed at high pickup pressure and is most noticeable at $11 \mathrm{~K}$ where the photoelectron signal is strongest. These fast electrons are attributed to the lower ionization energy of rare gas clusters. ${ }^{60}$ Figure 7 also provides a more global view of the trends exhibited by feature $\mathrm{C}$. At high electron energy, this feature extends to the sharp features A and B for both dopants; therefore, it is wider toward high energy in Xe-doped droplets. Below $4 \mathrm{eV}$, the shape of feature $\mathrm{C}$ is largely independent of dopant and depends only on source temperature.

In addition to the kinetic energy information, the angular distribution of the ejected electrons is extracted from the resulting photoelectron images. The angular distribution of the electrons has the form $I(\theta)=[1 /(4 \pi)]\left[1+\beta P_{2}(\cos \theta)\right],{ }^{61}$ where $\theta$ represents the angle between the axis of the polarization of the photon beam and the electron velocity vector and $\beta$ is the anisotropy parameter ranging between -1 and 2 with an isotropic distribution at $\beta=0$. Figure 8 shows the anisotropy parameter $\beta$ values for Xe-doped droplets derived from the whole image and from the individual features A, B, and C. An anisotropy value of 1.8 is derived from the atomic Xe image at $21.6 \mathrm{eV}$ (Figure 2c) and is shown as the dashed line in Figure 8 as reference. It can be seen from Figure 8 that the angular distributions of the ejected photoelectrons have been considerably altered by He droplets and are more isotropic than from bare Xe.

\section{Discussion}

Photoelectron images and the resulting photoelectron spectra of He droplets doped with $\mathrm{Kr}$ and $\mathrm{Xe}$ have revealed several intriguing features upon excitation at $21.6 \mathrm{eV}$. Features A and $\mathrm{B}$ each comprise a pair of peaks separated by the spin-orbit splitting of the dopant cation, with feature B shifted toward lower eKE by $0.65 \pm 0.02 \mathrm{eV}$ regardless of dopant. The relative ratio of features $\mathrm{A}$ and $\mathrm{B}$ depends on both source temperature and pickup cell pressure. The most prominent feature in the spectra, feature $\mathrm{C}$, is very broad, with its peak maximum moving toward higher eKE with decreasing source temperature. The shape of feature $\mathrm{C}$ is independent of dopant. Finally, there is feature $\mathrm{D}$, extremely slow electrons with $\sim 1 \mathrm{meV}$ kinetic 


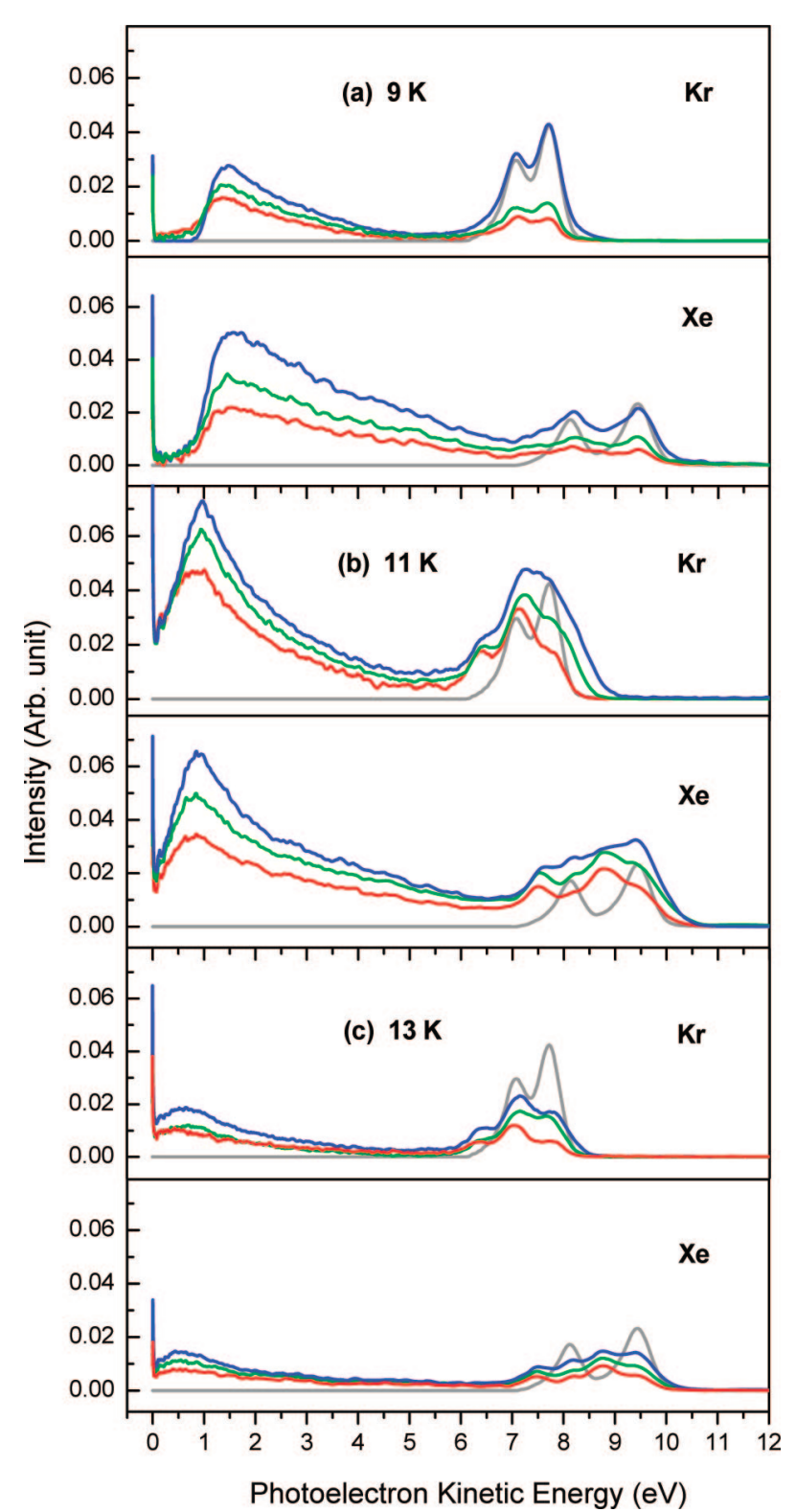

Figure 7. Photoelectron spectra of He droplets doped with $\mathrm{Kr}$ and $\mathrm{Xe}$ taken at (a) $9 \mathrm{~K}$, (b) $11 \mathrm{~K}$, and (c) $13 \mathrm{~K}$ at pickup pressures varying from $1.2 \times 10^{-5}$ to $3.3 \times 10^{-5}$ torr. Color code: red, low pickup pressure; green, medium pickup pressure; and blue, high pickup pressure. Note that the ultraslow peak (feature D) of Kr-doped droplets at $11 \mathrm{~K}$ is clipped for the ease of viewing all other features at different source temperatures within the same scale. Gray lines are from dopant atoms.

TABLE 2: Average Number of Dopant Captured by $\mathrm{He}$ Droplet at $T_{\mathrm{s}}=9,11$, and $13 \mathrm{~K}$, Calculated from Poisson Distribution

\begin{tabular}{lccc}
\hline & \multicolumn{3}{c}{$T_{\mathrm{s}}(\mathrm{K})$} \\
\cline { 2 - 4 } pickup pressure (torr) & 9 & 11 & 13 \\
\hline $1.2 \times 10^{-5}$ & 12 & 2 & 1 \\
$2.0 \times 10^{-5}$ & 19 & 3 & 2 \\
$3.3 \times 10^{-5}$ & 33 & 5 & 3
\end{tabular}

energy, similar to the feature observed previously in pure $\mathrm{He}$ droplets. ${ }^{25,39}$ In this section, we consider the various mechanisms responsible for each feature, noting that the nature of the electronic excitation of the He droplet, the dopant ionization mechanisms, and the interaction of the photoelectron with the droplet all play a role in determining the photoelectron image and spectrum.

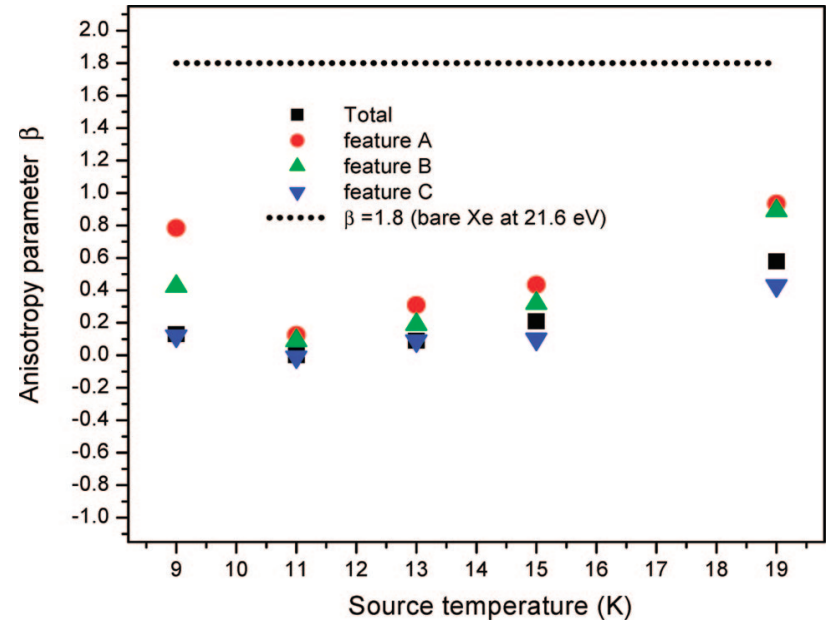

Figure 8. Anisotropy parameter $\beta$ of the whole image and features $\mathrm{A}, \mathrm{B}$, and $\mathrm{C}$ as a function of source temperature $T_{\mathrm{s}}$ at a pickup pressure of $5.5 \times 10^{-6}$ torr. The anisotropy parameter for the bare Xe at 21.6 $\mathrm{eV}, \beta=1.8$, is shown for reference as the dashed line.

A. Origins of Features A and B: Penning Ionization Pathways. Figure 3 shows that the energies of the two peaks comprising feature $\mathrm{A}$ in $\mathrm{Xe}$ - and $\mathrm{Kr}$-doped droplets match those for bare $\mathrm{Kr}$ and $\mathrm{Xe}$ at the same photon energy, $21.6 \mathrm{eV}$. We can rule out the possibility that feature A comes from bare atoms because if we look at the droplet photoelectron signal under the same conditions at $20.6 \mathrm{eV}$, where the droplets do not absorb, the intensity of the two peaks drops by over an order of magnitude. Moreover, as shown in Figure 8, the photoelectron angular distribution associated with feature A is markedly more isotropic than that for the bare atom. Hence feature A is indeed associated with excitation of doped droplets at $21.6 \mathrm{eV}$.

In pure $\mathrm{He}$ droplets, the excited electronic state at this energy ${ }^{36}$ is assigned to the analog of the $2 \mathrm{p}^{1} \mathrm{P}_{1}-1 \mathrm{~s}{ }^{1} \mathrm{~S}_{0}$ transition in atomic He at $21.22 \mathrm{eV}$. The blue-shift of $0.4 \mathrm{eV}$ in the droplet is attributed to the repulsive interaction of the He Rydberg electron in the excited $2 \mathrm{p}^{1} \mathrm{P}_{1}$ state with the droplet environment and can be thought of as the excess energy needed to form a Rydberg bubble state, in which the excitation is localized on a single atom, within the droplet. ${ }^{36,37,62}$ In pure He droplets, the excited $\mathrm{He}^{*}$ atom can be ejected from the droplet and fluoresce; this is how the electronic spectrum of pure He droplets was first measured. ${ }^{36}$ In doped He droplets, this excitation can also migrate to the dopant atom and ionize it via Penning ionization within the droplet, that is,

$$
\operatorname{He}^{*}\left(2 \mathrm{p}^{1} \mathrm{P}_{1}\right)\left[\mathrm{He}_{\mathrm{N}}\right]+\mathrm{Rg} \rightarrow \mathrm{He}\left[\mathrm{He}_{N}\right]+\mathrm{Rg}^{+}\left({ }^{2} \mathrm{P}_{3 / 2 \mathrm{p}, 1 / 2}\right)+\mathrm{e}^{-}
$$

Reaction 1 appears to compete effectively with Rydberg atom ejection, as discussed in previous work. ${ }^{24,26}$

The eKEs of the peaks comprising feature A show that the full excitation energy of $21.6 \mathrm{eV}$ is available for reaction 1 ; that is, the $0.4 \mathrm{eV}$ difference compared to the atomic transition is not dissipated in the droplet prior to dopant ionization. Instead, it is likely that a series of resonant excitation transfer collisions occurs within the droplet where the electronic excitation hops from one atom to another until a He atom adjacent to the dopant is excited, after which reaction 1 can proceed. This picture is similar to the resonant charge-hopping model invoked to explain electron impact ionization in doped He droplets. ${ }^{19}$

Feature B shows the same splitting as feature A, but both peaks are shifted to lower eKE by $0.65 \pm 0.02 \mathrm{eV}$, regardless 
TABLE 3: Excited State of Atomic He at $n=2$ with Corresponding Energy and Lifetime

\begin{tabular}{ccc}
\hline state & energy $(\mathrm{eV})$ & mean lifetime $(\mathrm{s})$ \\
\hline $2 \mathrm{p}{ }^{1} \mathrm{P}_{1}$ & 21.22 & $5.6 \times 10^{-10}$ \\
$2 \mathrm{p}{ }^{3} \mathrm{P}_{2}$ & 20.96 & $1.1 \times 10^{-7}$ \\
$2 \mathrm{~s}{ }^{1} \mathrm{~S}_{0}$ & 20.61 & $1.4 \times 10^{-1}$ \\
$2 \mathrm{~s}{ }^{3} \mathrm{~S}_{1}$ & 19.82 & $1.0 \times 10^{6}$
\end{tabular}

of the dopant. This observation suggests that feature B arises from relaxation of the initially excited droplet state to a lower excited-state prior to ionization of the dopant. Assuming that the shift is close to an energy level splitting in the $n=2$ manifold of atomic He, Table 3 suggests that the relaxation to droplet analogue of the $\mathrm{He} 2 \mathrm{~s}{ }^{1} \mathrm{~S}_{0}$ state is the most reasonable candidate because it lies $0.61 \mathrm{eV}$ below the $2 \mathrm{p}{ }^{1} \mathrm{P}_{1}$ state for atomic $\mathrm{He}$, close to the observed shift between features $\mathrm{A}$ and B. The small difference, $0.04 \mathrm{eV}$, between the shift of features $\mathrm{B}$ from feature $\mathrm{A}$ and the splitting of the atomic levels may reflect differing interactions of the Rydberg electron with the He droplet in these two states; the lower-lying $2 \mathrm{~s}{ }^{1} \mathrm{~S}_{0}$ is slightly smaller, with a radius of $1.10 \AA$ compared to $1.15 \AA$ for the $2 p$ ${ }^{1} \mathrm{P}_{1}$ state; ${ }^{63}$ therefore, it should experience a less repulsive interaction with the droplet.

Hence, we attribute feature B to a two-step process. First, the initially created $2 \mathrm{p}^{1} \mathrm{P}_{1}$ state relaxes to the $2 \mathrm{~s}{ }^{1} \mathrm{~S}_{0}$ state, most likely through inelastic excitation transfer collisions within the droplet,

$$
\left[\mathrm{He}^{*}\left(2 \mathrm{p}^{1} \mathrm{P}_{1}\right)+\mathrm{He}\right] \mathrm{He}_{\mathrm{N}} \rightarrow\left[\mathrm{He}^{*}\left(2 \mathrm{~s}{ }^{1} \mathrm{~S}_{0}\right)+\mathrm{He}\right] \mathrm{He}_{\mathrm{N}}
$$

followed by Penning ionization of the dopant,

$$
\mathrm{He}^{*}\left(2 \mathrm{~s}^{1} \mathrm{~S}_{0}\right)\left[\mathrm{He}_{\mathrm{N}}\right]+\mathrm{Rg} \rightarrow \mathrm{He}\left[\mathrm{He}_{\mathrm{N}}\right]+\mathrm{Rg}^{+}\left({ }^{2} \mathrm{P}_{3 / 2,1 / 2}\right)+\mathrm{e}^{-}
$$

For reaction 2 to be efficient in the very cold environment of a He droplet, there must be an attractive potential energy curve between the reactants that crosses a second curve leading to products. On the basis of the potential energy curves for excited states of $\mathrm{He}_{2} *$ constructed by Ginter et al., ${ }^{64}$ the likeliest candidates are the attractive $B^{1} \Pi_{\mathrm{g}}$ state, which correlates to $\mathrm{He}$ $+\mathrm{He}^{*}\left(2 \mathrm{p}{ }^{1} \mathrm{P}_{1}\right)$, and the $C^{1} \sum_{g}^{+}$state, which correlates to $\mathrm{He}+$ $\mathrm{He}^{*}\left(2 \mathrm{~s}{ }^{1} \mathrm{~S}_{0}\right)$, as illustrated in Figure 9 . The $C$ state is repulsive at long range and attractive at shorter range and crosses the $B$ state at $R \approx 2 \AA$. Note that many of the $\Sigma$ states of $\mathrm{He}_{2} *$ have barriers of varying size at large internuclear distances owing to repulsive orbital overlap interactions between the two atoms, ${ }^{63}$ but this effect is absent in the $\Pi$ states; therefore, they can be purely attractive. ${ }^{65}$

Our interpretation of features $\mathrm{A}$ and $\mathrm{B}$ requires that reactions 1 and 2 are of comparable efficiency in the droplet environment. Penning ionization (de-excitation) cross sections for $\mathrm{He} *\left(2 \mathrm{p}{ }^{1} \mathrm{P}_{1}\right)$ in collisions with $\mathrm{Kr}$ and $\mathrm{Xe}$ have been measured to be $7.2 \times$ $10^{-15}$ and $1.0 \times 10^{-14} \mathrm{~cm}^{2}$, respectively. ${ }^{66}$ To our knowledge, the rate of reaction 2 in binary collisions has not been reported, although the analogous process has been investigated within the $n=3$ manifold $^{67}$ and found to have a cross section of 4.5 $\times 10^{-16} \mathrm{~cm}^{2}$, a considerably lower number which is probably an upper bound, because the energy spacing within the $n=3$ manifold is considerably smaller. However, the highly dissipative environment in a He droplet may dramatically increase the efficiency of reaction 2 compared to an isolated binary collision. In a droplet, a collision between reactants on the $B$ state leads to highly vibrationally excited $\mathrm{He}_{2} *$. Given the very low energy of the collision, if only a small amount of this vibrational energy

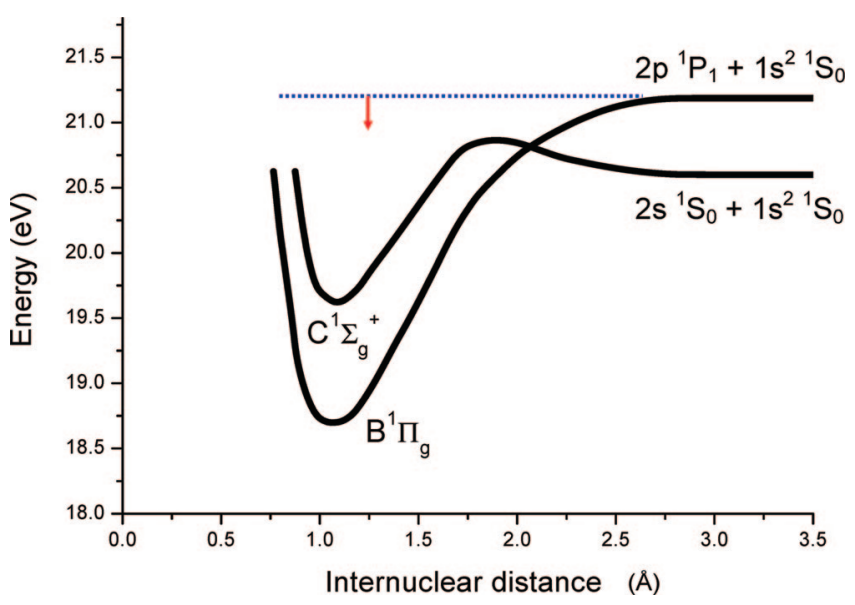

Figure 9. Potential energy curves for two excited states of $\mathrm{He}_{2}{ }^{*}, \mathrm{He}$ $+\mathrm{He} *\left(2 \mathrm{p}{ }^{1} \mathrm{P}_{1}\right)$, and $\mathrm{He}+\mathrm{He} *\left(2 \mathrm{~s}{ }^{1} \mathrm{~S}_{0}\right)$, constructed by Ginter et al. ${ }^{64}$

is lost to the droplet, dissociation to $\mathrm{He}+\mathrm{He}^{*}\left(2 \mathrm{p}{ }^{1} \mathrm{P}_{1}\right)$ will be closed, greatly increasing the interaction time and facilitating a nonadiabatic transition from the $B$ state to the $C$ state. Moreover, there is only a single (or a small number of) dopant atom(s) in a droplet comprising $\sim 10^{4} \mathrm{He}$ atoms; therefore, processes such as reaction 2, involving $\mathrm{He}-\mathrm{He}$ collisions, are far more likely to occur in a droplet than $\mathrm{He}-$ dopant collisions.

These considerations are consistent with the trends shown in Figure 5. For example, as the droplet size increases, we might expect $\mathrm{B} /(\mathrm{A}+\mathrm{B})$ to increase because it takes longer for the excitation on a $\mathrm{He}^{*}\left(2 \mathrm{p}{ }^{1} \mathrm{P}_{1}\right)$ atom to migrate to the dopant, whereas the rate of reaction 2 should either be relatively independent of cluster size or perhaps increase for larger droplets owing to their higher density (see Table 1). In fact, with the exception of the very large droplets formed at $T_{\mathrm{s}}=9 \mathrm{~K}$, this trend is borne out by the results shown in Figure 5. The exceptional behavior of the droplets at $9 \mathrm{~K}$ is likely caused by the very large coagulation cross section of He droplets formed at this temperature because of the transformation of the droplet formation mechanism from the subcritical to supercritical regime, ${ }^{59}$ these large droplets pick up many more dopants even at the lowest pickup cell pressures used in the current experiments (Table 2), possibly enhancing the cross section of reaction 1. The enhancement of reaction 1 by multiple dopant atoms is also consistent with the observation in Figure 7 that $\mathrm{B} /(\mathrm{A}+\mathrm{B})$ decreases with increasing pickup cell pressure.

B. Feature C: Inelastically Scattered Electrons. Because Penning ionization from excited $\mathrm{He}_{n}{ }^{*}$ states $\left(2 \mathrm{p}{ }^{1} \mathrm{P}_{1}\right.$ and $\left.2 \mathrm{~s}^{1} \mathrm{~S}_{0}\right)$ is believed to be the only mechanism by which electrons are generated in this experiment, the appearance of the much slower feature $\mathrm{C}$ indicates that some fraction of these electrons lose considerable kinetic energy prior to ejection from the droplet. This interpretation of feature $\mathrm{C}$ is consistent with the observation that (a) at high energy, it extends out to the sharp Penning features for both dopants and (b) its relative contribution grows with increasing droplet size, as shown in Figures 4 and 5. The photoelectron imaging experiments by Loginov et al. ${ }^{27}$ on aniline-doped droplets showed some evidence for inelastic scattering of electrons passing through the droplet, as did our prior work on droplets doped with $\mathrm{SF}_{6},{ }^{38}$ in neither case was this effect as evident as in the work presented here.

The shape of feature $\mathrm{C}$ is largely independent of dopant and pickup cell pressure, but it does depend strongly on cluster size, as shown in Figures 4 and 7. The peak maximum moves steadily toward higher eKE, and for the largest cluster size $(\langle N\rangle \approx$ 250000 , see Table 1), feature $\mathrm{C}$ exhibits a clear onset around 
$1 \mathrm{eV}$ with a maximum positioned at $1.45 \mathrm{eV}$. This last result is of particular interest because the observed onset coincides with the value $V_{0}=1.0 \mathrm{eV}$ of the conduction band edge for electrons in liquid He. $V_{0}$ has been well-established both experimentally ${ }^{45,47,48}$ and theoretically. ${ }^{68-70}$ It represents a barrier to injection of free electrons into bulk liquid $\mathrm{He}$ as well as the minimum kinetic energy that a free electron can have in the liquid before localization to a bubble state occurs. ${ }^{16}$

Electron scattering experiments on He droplets have also shown evidence for a barrier to electron injection and/or attachment. Martini et al. ${ }^{50}$ measured the cross section for electronic excitation of He droplets via electron scattering. They observed shifts of up to $1.5 \mathrm{eV}$ in the threshold energies needed to excite electronic states within the droplets when compared to bare $\mathrm{He}$ atoms and found this shift to be a strong function of density. Experiments by Henne and Toennies, ${ }^{51}$ in which they measured the energy dependence of the electron attachment yield to large He droplets comprising $10^{5}$ or more atoms, also showed evidence for a barrier in the $1 \mathrm{eV}$ range. In light of these observations, we interpret the $1 \mathrm{eV}$ onset of feature $\mathrm{C}$ for the largest droplets in the inverse sense, that is, as evidence for an escape barrier for electrons generated within the droplet. It thus appears that droplets in this size range are sufficiently large to support the droplet analogue of the conduction band in bulk liquid $\mathrm{He}$.

Although there is no obvious onset for the smaller droplet sizes, one can track the maximum position of feature $\mathrm{C}$ as the droplet size varies, as shown in Figure 6. From Figure 6, the peak of feature $\mathrm{C}$ becomes noticeable at a droplet radius of 24 $\pm 3 \AA(\langle N\rangle \approx 1200)$ and reaches a maximum value of $1.45 \mathrm{eV}$ at the largest droplet radius, regardless of dopant. Experiments and calculations on liquid and gas phase He have shown that $V_{0}$ drops significantly as the He density decreases from the bulk liquid $\mathrm{He}$ value of $2.2 \times 10^{22} \mathrm{~cm}^{-3}, 48,69$ dropping to about 0.5 $\mathrm{eV}$ at a density of $1 \times 10^{22} \mathrm{~cm}^{-3}$. These observations were used by Martini et al. to show that in He droplets, the density drops with decreasing cluster size, a trend confirmed in the more recent experiments by Harms et al., ${ }^{58}$ which are the basis for the droplet densities given in Table 1. Hence, assuming that the peak in feature $\mathrm{C}$ is related to the barrier height to electron escape, the trend in Figure 6 follows the expected dependence of $V_{0}$ on droplet density. On the other hand, the absence of an observable onset in feature $\mathrm{C}$ for the smaller clusters may indicate that (a) either the droplet is too small for the concept of a conduction band to be valid or (b) the attractive Coulomb potential between the electron and the positive charge in the droplet modifies the electron escape dynamics in the smaller clusters.

In any case, our overall interpretation of feature $\mathrm{C}$ is that it represents electrons formed from Penning ionization of the dopant by either unrelaxed or relaxed $\mathrm{He}^{*}$ that undergo significant energy loss while escaping from the droplet. The behavior of feature $\mathrm{C}$ at low eKEs is suggestive of a barrier associated with the conduction band edge of liquid He. We note that this barrier does not affect the energies of features A or B, presumably because the photoelectrons associated with these features escape by undergoing only a few elastic collisions with the droplet atoms.

The mechanism of electron energy loss responsible for feature $\mathrm{C}$ is of considerable interest. Our photoelectron spectra bear a superficial resemblance to the VUV photoemission spectra of rare-gas ( $\mathrm{Ar}, \mathrm{Kr}$, and $\mathrm{Xe}$ ) solid films measured by Schwentner, ${ }^{71}$ in that they show fast features from direct ionization of the rare gas atoms and much slower features at low eKE. In those spectra, the slow features were attributed to inelastic electron-electron scattering, because the electrons produced by direct ionization had sufficient kinetic energy $(>12 \mathrm{eV})$ to create electronic excitations in the surrounding medium. Such a mechanism is not available in $\mathrm{He}$ droplets under the conditions of our experiment, because the maximum eKE created by Penning ionization, around $10 \mathrm{eV}$, is substantially below the lowest electronic excitation of the droplet at $21.6 \mathrm{eV}$. The question of whether the superfluid nature of He droplets enhances electron energy loss is an open one, and although it lies beyond the scope of this paper, it is certainly worthy of further consideration.

C. Feature D: Ultraslow Electrons. Feature D, from ultraslow electrons, appears as a small bright central spot in all the photoelectron images except at the highest temperatures reported, $19 \mathrm{~K}$, where there is relatively little droplet formation. It appears to be the same feature that was seen in our photoelectron images of pure He droplets. At $23.5 \mathrm{eV},{ }^{25}$ an energy below the ionization potential of atomic $\mathrm{He}(24.6 \mathrm{eV})$ but above the ionization threshold of $\mathrm{He}$ droplets $(\sim 23 \mathrm{eV}),{ }^{24}$ this was the only electron signal seen. It was also present in our photoelectron images of pure droplets above $24.6 \mathrm{eV}$, which were dominated by fast electrons from direct ionization of $\mathrm{He}$ atoms within the droplet. ${ }^{39}$ In that work, the ultraslow electrons disappeared at higher source temperatures $(>17 \mathrm{~K})$ under conditions where there was still a considerable fast electron signal from ionization of atomic He. Hence, these ultraslow electrons appear in all images in which either pure or doped droplet photoionization occurs, and they disappear only under conditions where droplet formation is greatly reduced (or eliminated).

As mentioned in the Introduction, the possible origin of feature D has been considered at length in our previous investigations of pure droplets. ${ }^{25,39} \mathrm{We}$ proposed that this feature arises from electrons that drop below the conduction band edge and thus become localized in electron bubble states. The formation of bubble states in bulk liquid He is well-established, ${ }^{15,43,44,46,72}$ where they have a radius of $\sim 17 \AA$ with an effective mass of $243{ }^{4} \mathrm{He}$, and there is considerable experimental and theoretical support for their existence in $\mathrm{He}$ droplets. ${ }^{14,52,73-75}$ If the bubble migrates to the droplet surface, the electron can escape by tunneling over a low barrier, calculated to be only $3 \mathrm{meV}$ at the surface of bulk liquid He. ${ }^{76}$ It may also get trapped at the surface in a diffuse Rydberg state of the type originally considered by Golov and Sekatskii ${ }^{40}$ and more recently by Ancilotto et al. ${ }^{41}$ Once this occurs, the electron is ejected with very low kinetic energy by vibrational autoionization, in which low-frequency droplet modes excited by the formation of a positive charge within the droplet couple to the weakly bound electron. The results presented here offer little new insight into feature $\mathrm{D}$, other than to demonstrate that it can be produced by yet another mechanism, in this case Penning ionization of a rare gas dopant.

\section{Conclusion}

The photoionization and photoelectron dynamics of $\mathrm{He}$ droplets doped with $\mathrm{Kr}$ and $\mathrm{Xe}$ atoms were investigated by excitation with VUV synchrotron radiation and photoelectron imaging. Our experiments focused on indirect dopant ionization by exciting the droplets at $21.6 \mathrm{eV}$, corresponding to an intense band in the electronic spectrum of pure He droplets that lies below their ionization threshold. The dopant atoms are then ionized by an intracluster Penning mechanism. The resulting photoelectron images and spectra show how both the kinetic energy and angular distributions of ejected 
photoelectrons are affected by the droplet environment. More specifically, we observed four distinct mechanisms for photoelectron ejection.

Sharp pairs of features from two Penning ionization channels are identified: one from $\mathrm{He}^{*}\left(2 \mathrm{p}{ }^{1} \mathrm{P}_{1}\right)+\mathrm{Rg}$ (feature A) and one from $\mathrm{He}^{*}\left(2 \mathrm{~s}{ }^{1} \mathrm{~S}_{0}\right)+\mathrm{Rg}$ (feature B). Feature A originates from the initial, unrelaxed excitation of the droplet at $21.6 \mathrm{eV}$, whereas feature $\mathrm{B}$ is attributed to relaxation of the $\mathrm{He}^{*}$ from the $2 \mathrm{p}^{1} \mathrm{P}_{1}$ to $2{ }^{2} \mathrm{~S}_{0}$ state via $\mathrm{He}-\mathrm{He}^{*}$ collisions within the droplet, followed by Penning ionization of the dopant. However, the photoelectrons associated with both features show no evidence for energy exchange with the droplet atoms as they escape.

In contrast, the broad, unstructured peak seen in all the spectra (feature C) is attributed to electrons produced by the above Penning processes that undergo significant energy loss in transit through the droplet. Moreover, for the largest droplets considered, with $\langle N\rangle \approx 250000$, this feature shows an onset around $1 \mathrm{eV}$, which coincides with the conduction band edge, $V_{0}$, in liquid He. This agreement suggests that the conduction band edge acts as an exit barrier with respect to the vacuum for electrons leaving the droplet, just as it acts as an entrance barrier for the injection of electrons into the droplet. Finally, we also observe a small amount of ultraslow electrons with kinetic energy peaking around $1 \mathrm{meV}$, similar to our earlier work on photoelectron imaging of pure He droplets.

There are many fundamental questions raised by this study, including (i) the exact nature of the excited droplet state at 21.6 $\mathrm{eV}$, (ii) the time scale for Penning ionization of the dopant subsequent to droplet excitation, (iii) the efficiency of the relaxation process invoked to explain feature $B$, (iv) the extent to which electrons escape the droplet cleanly versus losing substantial energy during their escape, (v) the role of the conduction band edge in the kinetic energy distributions and how its effects vary with cluster size, and (vi) the role of the positive charge created by ionization on the photoelectron dynamics, that is, how the interactions between an electron and charged droplet differ from those with a neutral droplet or with bulk liquid $\mathrm{He}$. All of these issues should be attractive if somewhat complex targets for theoretical investigations; such work would aid and clarify the interpretation of the experimental results presented here.

Acknowledgment. This work was supported by the Director, Office of Science, Office of Basic Energy Sciences, Chemical Sciences Division of the U.S. Department of Energy under Contract no. DE-AC02-05CH11231.

\section{References and Notes} 54,31

(1) Toennies, J. P.; Vilesov, A. F.; Whaley, K. B. Physics Today 2001 2083.

(2) Grebenev, S.; Toennies, J. P.; Vilesov, A. F. Science 1998, 279,

(3) Sindzingre, P.; Klein, M. L.; Ceperley, D. M. Phys. Rev. Lett. 1989, 63,1601 .

(4) Goyal, S.; Schutt, D. L.; Scoles, G. Phys. Rev. Lett. 1992, 69, 933.

(5) Scheidemann, A.; Schilling, B.; Toennies, J. P.; Northby, J. A. Physica B 1990, 165, 135 .

(6) Hartmann, M.; Miller, R. E.; Toennies, J. P.; Vilesov, A. F. Science 1996, $272,1631$.

(7) Northby, J. A. J. Chem. Phys. 2001, 115, 10065.

(8) Callegari, C.; Lehmann, K. K.; Schmied, R.; Scoles, G. J. Chem. Phys. 2001, 115, 10090.

(9) Stienkemeier, F.; Vilesov, A. F. J. Chem. Phys. 2001, 115, 10119 2622.

(10) Toennies, J. P.; Vilesov, A. F. Angew. Chem., Int. Ed. 2004, 43,

(11) Higgins, J.; Callegari, C.; Reho, J.; Stienkemeier, F.; Ernst, W. E.; Lehmann, K. K.; Gutowski, M.; Scoles, G. Science 1996, 273, 629.

(12) Nauta, K.; Miller, R. E. Science 1999, 283, 1895.
(13) Nauta, K.; Miller, R. E. Science 2000, 287, 293.

(14) Jiang, T.; Kim, C.; Northby, J. A. Phys. Rev. Lett. 1993, 71, 700.

(15) Northby, J. A.; Sanders, T. M. Phys. Rev. Lett. 1967, 18, 1184.

(16) Rosenblit, M.; Jortner, J. Phys. Rev. Lett. 1995, 75, 4079.

(17) Buchenau, H.; Toennies, J. P.; Northby, J. A. J. Chem. Phys. 1991, 95,8134

(18) Jiang, T.; Northby, J. A. Phys. Rev. Lett. 1992, 68, 2620.

(19) Callicoatt, B. E.; Forde, K.; Ruchti, T.; Jung, L. L.; Janda, K. C.; Halberstadt, N. J. Chem. Phys. 1998, 108, 9371.

(20) Ruchti, T.; Forde, K.; Callicoatt, B. E.; Ludwigs, H.; Janda, K. C. J. Chem. Phys. 1998, 109, 10679.

(21) Yang, S. F.; Brereton, S. M.; Wheeler, M. D.; Ellis, A. M. Phys. Chem. Chem. Phys. 2005, 7, 4082.

(22) Denifl, S.; Stano, M.; Stamatovic, A.; Scheier, P.; Mark, T. D. J. Chem. Phys. 2006, 124, 054320.

(23) Ren, Y.; Kresin, V. J. Chem. Phys. 2008, 128, 074303.

(24) Fröchtenicht, R.; Henne, U.; Toennies, J. P.; Ding, A.; FieberErdmann, M.; Drewello, T. J. Chem. Phys. 1996, 104, 2548.

(25) Peterka, D. S.; Lindinger, A.; Poisson, L.; Ahmed, M.; Neumark, D. M. Phys. Rev. Lett. 2003, 91, 043401.

(26) Kim, J. H.; Peterka, D. S.; Wang, C. C.; Neumark, D. M. J. Chem. Phys. 2006, 124, 214301.

(27) Loginov, E.; Rossi, D.; Drabbels, M. Phys. Rev. Lett. 2005, 95, 163401.

(28) Loginov, E.; Drabbels, M. J. Phys. Chem. A 2007, 111, 7504.

(29) Radcliffe, P.; Przystawik, A.; Diederich, T.; Doppner, T.; Tiggesbaumker, J.; Meiwes-Broer, K. H. Phys. Rev. Lett. 2004, 92, 173403.

(30) Polyakova, E.; Stolyarov, D.; Wittig, C. J. Chem. Phys. 2006, 124 , 214308.

(31) Curtis, S.; Boatwright, A.; Wright, R. R.; Stace, A. J. Chem. Phys. Lett. 2005, 401, 254.

(32) Brindle, C. A.; Prado, M. R.; Janda, K. C.; Halberstadt, N.; Lewerenz, M. J. Chem. Phys. 2005, 123, 064312.

(33) Bonhommeau, D.; Lake, P. T.; Le Quiniou, C.; Lewerenz, M.; Halberstadt, N. J. Chem. Phys. 2007, 126, 051104.

(34) Bonhommeau, D.; Halberstadt, N.; Viel, A. J. Chem. Phys. 2006, 124,184314

(35) Scifoni, E.; Gianturco, F. A. Eur. Phys. J. D 2002, 21, 323.

(36) Joppien, M.; Karnbach, R.; Moller, T. Phys. Rev. Lett. 1993, 71, 2654.

(37) von Haeften, K.; Laarmann, T.; Wabnitz, H.; Moller, T. J. Phys. B 2005, 38, S373.

(38) Peterka, D. S.; Kim, J. H.; Wang, C. C.; Neumark, D. M. J. Phys. Chem. B 2006, 110, 19945.

(39) Peterka, D. S.; Kim, J. H.; Wang, C. C.; Poisson, L.; Neumark, D. M. J. Phys. Chem. A 2007, 111, 7449.

(40) Golov, A.; Sekatskii, S. Z. Phys. D 1993, 27, 349.

(41) Ancilotto, F.; Pi, M.; Mayol, R.; Barranco, M.; Lehmann, K. K. J. Phys. Chem. A 2007, 111, 12695.

(42) Przystawik, A.; Radcliffe, P.; Diederich, T.; Doppner, T.; Tiggesbaumker, J.; Meiwes-Broer, K. H. J. Chem. Phys. 2007, 126, 184306.

(43) Rice, S. A. Acc. Chem. Res. 1968, $1,81$.

(44) Hernandez, J. P.; Silver, M. Phys. Rev. A 1970, 2, 1949.

(45) Sommer, W. T. Phys. Rev. Lett. 1964, 12, 271.

(46) Fowler, W. B.; Dexter, D. L. Phys. Rev. 1968, 176, 337

(47) Woolf, M. A.; Rayfield, G. W. Phys. Rev. Lett. 1965, 15, 235.

(48) Asaf, U.; Steinberger, I. T. Chem. Phys. Lett. 1986, 128, 91.

(49) Schmidt, W. F.; Illenberger, E.; Khrapak, A. G.; Sakai, Y.; Yoshino,

K. IEEE Trans. Dielectr. Electr. Insul. 2003, 10, 1012.

(50) Martini, K.; Toennies, J. P.; Winkler, C. Chem. Phys. Lett. 1991, $178,429$.

(51) Henne, U.; Toennies, J. P. J. Chem. Phys. 1998, 108, 9327.

(52) Rosenblit, M.; Jortner, J. J. Chem. Phys. 2006, 124, 194505.

(53) Lewerenz, M.; Schilling, B.; Toennies, J. P. Chem. Phys. Lett. 1993, 206,381 .

(54) Lewerenz, M.; Schilling, B.; Toennies, J. P. J. Chem. Phys. 1995, 102,8191 .

(55) Eppink, A.; Parker, D. H. Rev. Sci. Instrum. 1997, 68, 3477.

(56) Dribinski, V.; Ossadtchi, A.; Mandelshtam, V. A.; Reisler, H. Rev. Sci. Instrum. 2002, 73, 2634

(57) Kuyatt, C. E.; Simpson, J. A.; Mielczarek, S. R. Phys. Rev. 1965, $138, \mathrm{~A} 385$.

(58) Harms, J.; Toennies, J. P.; Dalfovo, F. Phys. Rev. B 1998, 58, 3341. (59) Buchenau, H.; Knuth, E. L.; Northby, J.; Toennies, J. P.; Winkler, C. J. Chem. Phys. 1990, 92, 6875.

(60) Gantefor, G.; Broker, G.; Holub-Krappe, E.; Ding, A. J. Chem. Phys. 1989, 91, 7972.

(61) Yang, C. N. Phys. Rev. 1948, 74, 764.

(62) vonHaeften, K.; deCastro, A. R. B.; Joppien, M.; Moussavizadeh, L.; vonPietrowski, R.; Moller, T. Phys. Rev. Lett. 1997, 78, 4371.

(63) Guberman, S. L.; Goddard, W. A. Phys. Rev. A 1975, 12, 1203.

(64) Ginter, M. L.; Battino, R. J. Chem. Phys. 1970, 52, 4469.

(65) Guberman, S. L.; Goddard, W. A. Chem. Phys. Lett. 1972, 14, 460. 
(66) Ukai, M.; Nakazawa, H.; Shinsaka, K.; Hatano, Y. J. Chem. Phys. 1988, $88,3623$.

(67) Wellenstein, H. F.; Robertson, W. W. J. Chem. Phys. 1972, 56, 1072 .

(68) Kestner, N. R.; Jortner, J.; Cohen, M. H.; Rice, S. A. Phys. Rev. 1965, 140, A56.

(69) Plenkiewicz, B.; Plenkiewicz, P.; Jay-Gerin, J. P. Chem. Phys. Lett. 1989, $163,542$.

(70) Rosenblit, M.; Jortner, J. J. Phys. Chem. A 1997, 101, 751.

(71) Schwentner, N. Phys. Rev. B 1976, 14, 5490.
(72) Eloranta, J.; Schwentner, N.; Apkarian, V. A. J. Chem. Phys. 2002, 116, 4039 .

(73) Farnik, M.; Toennies, J. P. J. Chem. Phys. 2003, 118, 4176.

(74) Farnik, M.; Samelin, B.; Toennies, J. P. J. Chem. Phys. 1999, 110, 9195.

(75) Farnik, M.; Henne, U.; Samelin, B.; Toennies, J. P. Phys. Rev. Lett. 1998, 81,3892 .

(76) Ancilotto, F.; Toigo, F. Phys. Rev. B 1994, 50, 12820.

JP802332F 